\title{
Cerebral Lactate Correlates with Early Onset Pneumonia after Aneurysmal SAH
}

\author{
S. Radolf • N. Smoll • C. Drenckhahn • J. P. Dreier • \\ P. Vajkoczy • A. S. Sarrafzadeh
}

Received: 15 August 2013 / Revised: 23 September 2013 / Accepted: 26 September 2013 / Published online: 17 October 2013

(C) Springer Science+Business Media New York 2013

\begin{abstract}
Pneumonia is a significant medical complication following aneurysmal subarachnoid hemorrhage (aSAH). The aSAH may initiate immune interactions leading to depressed immunofunction, followed by an increased risk of infection. It remains unclear as to whether there is a possible association between cerebral metabolism and infections. Clinical and microdialysis data from aSAH patients prospectively included in the CoOperative Study on Brain Injury Depolarisations protocol Berlin were analyzed. Levels of glucose, lactate, pyruvate, and glutamate were measured hourly using microdialysis in the cerebral extracellular fluid. The occurrence of pneumonia (defined by positive microbiological cultures) and delayed ischemic neurological deficits (DIND) was documented prospectively. Eighteen aSAH patients ( $52.7 \pm 10.7$ years), classified according to the World Federation of Neurological Surgeons in low (I-III, $n=9$ ) and high (IV-V, $n=9)$ grades, were studied. Eight patients
\end{abstract}

S. Radolf $\cdot$ P. Vajkoczy $\cdot$ A. S. Sarrafzadeh

Department of Neurosurgery, Charité - Universitätsmedizin Berlin, Berlin, Germany

N. Smoll

Gippsland Medical School, Monash University,

Melbourne, Australia

C. Drenckhahn · J. P. Dreier

Center for Stroke Research Berlin, Berlin, Germany

C. Drenckhahn · J. P. Dreier

Department of Neurology, Charité - Universitätsmedizin Berlin,

Berlin, Germany

\section{J. P. Dreier}

Departments of Experimental Neurology and Neurosurgery, Department of Neurology, Charité - Universitätsmedizin Berlin, Berlin, Germany

\section{A. S. Sarrafzadeh $(\square)$}

Division of Neurosurgery, Geneva University Hospitals,

Geneva Neuroscience Center, Faculty of Medicine,

University of Geneva, Geneva, Switzerland

e-mail: asita.sarrafzadeh@hcuge.ch
(45\%) experienced DIND, 10 patients (56\%) pneumonia (mean onset day 2.6). Lactate was elevated at day 3 in infected patients $(n=9$, median $=6.82 \mathrm{mmol} / \mathrm{L})$ vs. patient without infections $(n=6$, median $=2.90 \mathrm{mmol} / \mathrm{L}, p=0.036)$. The optimum cut-off point to predict pneumonia at day 3 was $3.57 \mathrm{mmol} / \mathrm{L}$ with a sensitivity of 0.77 , and a specificity of 0.66 (area under curve was 0.833 with $p=0.034$ ). Lactate at day 7 was higher in DIND patients compared to no-DIND-patients $(p=0.016)$. Early elevated lactate correlated with occurrence of bacterial pneumonia, while late elevations with DIND after aSAH. Future investigations may elucidate the relationship between cerebral lactate and markers of immunocompetence and more detailed to identify patients with higher susceptibility for infections.

Keywords Microdialysis $\cdot$ Lactate $\cdot$ Aneurysmal Subarachnoid Hemorrhage $\cdot$ Pneumonia

\section{Introduction}

Cerebral lactate in extracellular fluid (ECF) is frequently high after aneurysmal subarachnoid hemorrhage (aSAH) in patients with delayed ischemic deficits presumably due to the presence of anaerobic glycolysis as a result of ischemia. Furthermore, the presence of lactate in the ECF is known to be associated with unfavorable outcome after aSAH [1]

In aSAH, pneumonia is described in $20 \%$ of the patients and has been associated with a threefold increased risk of death at 30 days for stroke patients $[2,3]$. Therefore, the occurrence of systemic infection after aSAH may be a symptom of impaired immune competence in these patients [4-6].

There is a rising debate about the role of inflammation for the progression of aSAH-induced secondary brain damage and complications after SAH [7, 8]. Several studies have confirmed the activation of the immune system with a prominent elevation of interleukin (IL)-6, IL-1 beta, and TNF-alpha early after aSAH [9]. Importantly, intracerebral 
IL-6 values were predictive for the development of secondary brain injury, delayed ischemic events, and critical brain swelling $[10-15]$. It is postulated that aSAH turns the cerebrospinal fluid (CSF) and cerebral extracellular fluid into a proinflammatory milieu [16]. The inflammatory activation of the immune system followed by an immunodepressive counter-reaction in aSAH patients might be an important contributor to secondary brain injury.

Preliminary data have shown that immunodepression with increased susceptibility for infections exist early and remains prolonged among aSAH patients with acute focal deficits (AFND), or delayed ischemic neurological deficit (DIND) [6]. In these patients, a persistent suppression of the cellular immune response as well as an impaired pro-inflammatory cytokine release by monocytes and lymphocytes throughout the observation period of 10 days was observed, while in asymptomatic patients (no DIND), parameters of immune competence recovered within 3-5 days after aSAH [6].

This study aims to explore an association between markers of cerebral metabolism measured in brain interstitial fluid of aSAH patients (reflecting the primary damage after $\mathrm{SAH}$ ), and the development of bacterial infections.

\section{Materials and Methods}

\section{Patient Population}

Between October 2010, and January 2012, 18 consecutive aSAH patients from the university hospital of Charité Berlin were enrolled in a prospective study on cerebral metabolism in the CoOperative Study on Brain Injury Depolarisations protocol. The local Research Ethics Committee approved this study in compliance with the Declaration of Helsinki. Written informed consent was obtained from the patients or nearest family relatives.

Clinical presentation was graded according to the World Federation of Neurological Surgeons (WFNS) [17] and patients were categorized according to the WFNS score as low grade (WFNS score I-III) or high grade (WFNS score IV-V) SAH patients and the Hunt and Hess grading system [18]. Aneurysm location was assessed using four-vessel angiography and/or CT angiography on the day of admission. The distribution and pattern of the hemorrhage was graded as proposed by Fisher [19]. The details and the management of patients with aSAH in the intensive care unit have been described elsewhere (http://www.cosbid.org).

\section{Diagnosis of Delayed Ischemic Neurological deficits}

A DIND was defined as the occurrence of focal neurological impairment (such as hemiparesis, aphasia, apraxia, hemianopia, or neglect) or a decrease of at least 2 points on the Glasgow Coma Scale [either on the total score or on one of its individual components (eye, motor on either side, verbal)] [20-22].

Moreover, the diagnosis of a DIND required that the neurological deficit was not present immediately after aneurysm occlusion, lasted for at least $1 \mathrm{~h}$, and could not be attributed to other causes such as hydrocephalus or rebleeding by means of clinical assessment, CT or MRI of the brain, and appropriate laboratory studies. Neuroimaging studies were performed postoperatively, at the time of clinical deterioration and after the monitoring period to screen for delayed infarcts.

\section{Bedside Microdialysis}

A microdialysis catheter (CMA 70, CMA, Sweden; length $10 \mathrm{~mm}$, cut-off $100 \mathrm{kDa}$ ) was inserted after aneurysm clipping into brain parenchyma of the respective vascular territory of the aneurysm. Insertion depth of the catheter tip was approximately $15 \mathrm{~mm}$ from dura level. Location of the catheter tip was controlled on postoperative CT scan. The catheter was perfused with sterile Ringer's solution at a flow rate of $0.3 \mu \mathrm{l} / \mathrm{min}$. On the outlet tube, perfusates were collected in microvials, exchanged hourly and stored at $-80{ }^{\circ} \mathrm{C}$, as previously described [9]. Average median values $(12 \mathrm{~h})$ were analyzed for patients with AFND, pneumonia, and DIND. Microdialysis started within the first $24 \mathrm{~h}$ after admission.

\section{Adverse Events}

Adverse events have been recorded prospectively according to the published criteria: [23] Pneumonia: new infiltrate on chest $\mathrm{x}$-ray, with purulent sputum, and/or positive microbiological cultures. Delayed ischemic neurological deficits: see above. AFND based on the clinical course, with symptoms, such as hemiparesis, or aphasia related to the initial hemorrhage with corresponding $\mathrm{CT}$ findings.

\section{Data Analysis}

Descriptive Statistics and Univariate Analysis

Intergroup comparisons were performed on daily assessed values for microdialysis (MD) parameters within the first 12 days after aSAH using the Mann-Whitney $U$ test for two samples. Fisher's exact test was used to test for group-specific differences in patient's characteristics. MD parameter concentration was expressed as median [inter-quartile range]. 
Multivariate Analysis of Variance

Performing multivariate analysis of variance (MANOVA) allows a simultaneous comparison of the medians of lactate levels across the dichotomized clinical groups of this study, taking into account their co-variation - as represented by the error variance-covariance matrix in the MANOVA model. Because the independent clinical variables in our study comprises more than two groups, we focused on MANOVA using Pillai's trace [24] since this method provides a multivariate tests that is robust to differences among the error variancecovariance matrices within groups, as well as to departures from the assumption of multivariate normality [25]. In addition, a receiver operating characteristic curve (ROC) was constructed for levels of lactate at days with significant different median levels to determine cut-off values, specificity, and sensitivity. All statistical analyses were performed with PASW Statistics release 18 (SPSS Inc., Chicago, IL, USA). Differences were considered statistically significant at $p<0.05$.

\section{Results}

\section{Patient Characteristics}

Demographic data of the 18 patients are summarized in Tables 1 und 2. Eighteen aSAH patients $(52.7 \pm 10.7$ years; mean \pm standard deviation) have been studied, and classified according to the WFNS in low grade (I-III, $n=9$ ) and high grade (IV-V, $n=9$ ). Patient groups (developing with or with no pneumonia) were comparable for age, gender, and duration of microdialysis monitoring. There were no differences in patient characteristics for patients with or without DIND (data not shown).

Non-infectious and Infectious Adverse Events after Aneurysmal SAH

Six patients (33\%) presented AFND. Eight patients (45\%) developed DIND (onset day median $=7$; range $=5-15$ ) (Fig. 1). Ten patients (56\%) suffered from early onset pneumonia (onset day median $=2.5$; range $=1-4$ ) as first infection. Five of them developed additional infections: one with urinary tract infection at day 13 , one with meningitis/ventriculitis at day 9, two with septicemia at days 4 and 11, and one with sinusitis. Pathogens isolated in patients with pneumonia were as follows: Pseudomonas aeroginosa $(n=3)$, Klebsiella oxytoca $(n=1)$, Klebsiella pneumoniae $(n=1)$, Enterobacter aerogenes $(n=1)$, Serratia marcescens $(n=1)$. In three cases, only the radiological and clinical criteria of pneumonia were fulfilled. Nearly $90 \%$ of the patients had been ventilated mechanically. In this study, a relation between ventilation and incidence of pneumonia could not be proven. Also, the mechanical ventilation durations of patients without/with AFND and without/with DIND did not differ significantly. Fig. 1 demonstrates the baseline

Table 1 Patient characteristics for pneumonia

\begin{tabular}{|c|c|c|c|c|}
\hline & All patients $(n=18)$ & No pneumonia $(n=8)$ & Pneumonia $(n=10)$ & $p$ Value \\
\hline Age & 51.5 [31-77] & $54[48-62]$ & $56[41-63]$ & NS \\
\hline Male/female & $8 / 10(55 / 45 \%)$ & $4 / 4(50 / 50 \%)$ & $4 / 6(40 / 60 \%)$ & NS \\
\hline Coiling/clipping & $5 / 13(28 / 72 \%)$ & $4 / 4(50 / 50 \%)$ & $1 / 9(10 / 90 \%)$ & 0.088 \\
\hline AFND & $6(33 \%)$ & $2(25 \%)$ & $4(40 \%)$ & NS \\
\hline DIND & $8(44 \%)$ & $5(62 \%)$ & $3(33 \%)$ & NS \\
\hline Hunt and Hess grade & $3[1-5]$ & $3[1-4]$ & $3.5[1-5]$ & NS \\
\hline WFNS grade & $3[1-5]$ & $3[1-5]$ & $3.5[1-5]$ & NS \\
\hline Grade I & $4(22.2 \%)$ & $1(12.5 \%)$ & $3(30 \%)$ & \\
\hline Grade II & $4(22.2 \%)$ & $3(37.5 \%)$ & $1(10 \%)$ & \\
\hline Grade III & $1(6 \%)$ & $0(0 \%)$ & $1(10 \%)$ & \\
\hline Grade IV & $6(33.3 \%)$ & $3(37.5 \%)$ & $3(30 \%)$ & \\
\hline Grade V & $2(16.7 \%)$ & $1(12.5 \%)$ & $2(20 \%)$ & \\
\hline Fisher score & $3[1-4]$ & $3[1-4]$ & $3[3-3]$ & \\
\hline Extended Glasgow outcome score (EGOS) after 180 days & $2[0-7]$ & $2[0-7]$ & $1[0-7]$ & \\
\hline Modified ranking scale after 15 days & $5[0-6]$ & $5[0-6]$ & $5[3-6]$ & NS \\
\hline Mortality 180 days & $6(33.3 \%)$ & $2(25 \%)$ & $4(40 \%)$ & NS \\
\hline Duration of mechanical ventilation (hrs) & $226[33-357]$ & $268[63-331]$ & $178[0-357]$ & NS \\
\hline
\end{tabular}

Data are expressed as median [min-max] or as absolute numbers

$A F N D$ acute focal neurological deficit, DIND delayed ischemic neurological deficit, WFNS World Federation of Neurological Surgeons 
Table 2 Patient characteristics for DIND

\begin{tabular}{|c|c|c|c|c|}
\hline & All patients $(n=18)$ & No DIND $(n=10)$ & $\operatorname{DIND}(n=8)$ & $p$ Value \\
\hline Age & 51.5 [31-77] & $49[31-68]$ & $54[36-77]$ & NS \\
\hline Male/female & $8 / 10(55 / 45 \%)$ & $4 / 6(40 / 60 \%)$ & $4 / 4(50 / 50 \%)$ & NS \\
\hline Coiling/clipping & $5 / 13(28 / 72 \%)$ & $3 / 7(30 / 70 \%)$ & $2 / 6(25 / 75 \%)$ & NS \\
\hline AFND & $6(33 \%)$ & $2(25 \%)$ & $4(40 \%)$ & NS \\
\hline Hunt and Hess grade & $3[1-5]$ & $3(1-5)$ & $3.5(2-4)$ & NS \\
\hline WFNS grade & $3[1-5]$ & $2(1-5)$ & $4(1-5)$ & NS \\
\hline Grade I & $4(22.2 \%)$ & $3(37.5 \%)$ & $1(10 \%)$ & \\
\hline Grade II & $4(22.2 \%)$ & $2(25 \%)$ & $2(20 \%)$ & \\
\hline Grade III & $1(6 \%)$ & $0(0 \%)$ & $1(10 \%)$ & \\
\hline Grade IV & $6(33.3 \%)$ & $1(12.5 \%)$ & $5(50 \%)$ & \\
\hline Grade V & $2(16.7 \%)$ & $2(25 \%)$ & $1(10 \%)$ & \\
\hline Fisher score & $3[1-4]$ & $3[1-4]$ & $3[3-4]$ & NS \\
\hline Extended Glasgow outcome score (EGOS) after 180 days & $2[0-7]$ & $4[0-7]$ & $1[0-7]$ & NS \\
\hline Modified ranking scale after 15 days & $5[0-6]$ & $5[0-6]$ & $5[1-6]$ & NS \\
\hline Mortality 180 days & $6(33.3 \%)$ & $2(25 \%)$ & $4(40 \%)$ & NS \\
\hline Duration of mechanical ventilation $(\mathrm{h})$ & $226[33-357]$ & $239[33-331]$ & $307[80-357]$ & NS \\
\hline
\end{tabular}

Data are expressed as median [min-max] or as absolute numbers

$A F N D$ acute focal neurological deficit, DIND delayed ischemic neurological deficit, WFNS World Federation of Neurological Surgeons

differences between patients with and without pneumonia. The median duration of mechanical ventilation was less in patients with pneumonia, though this difference did not reach significance.

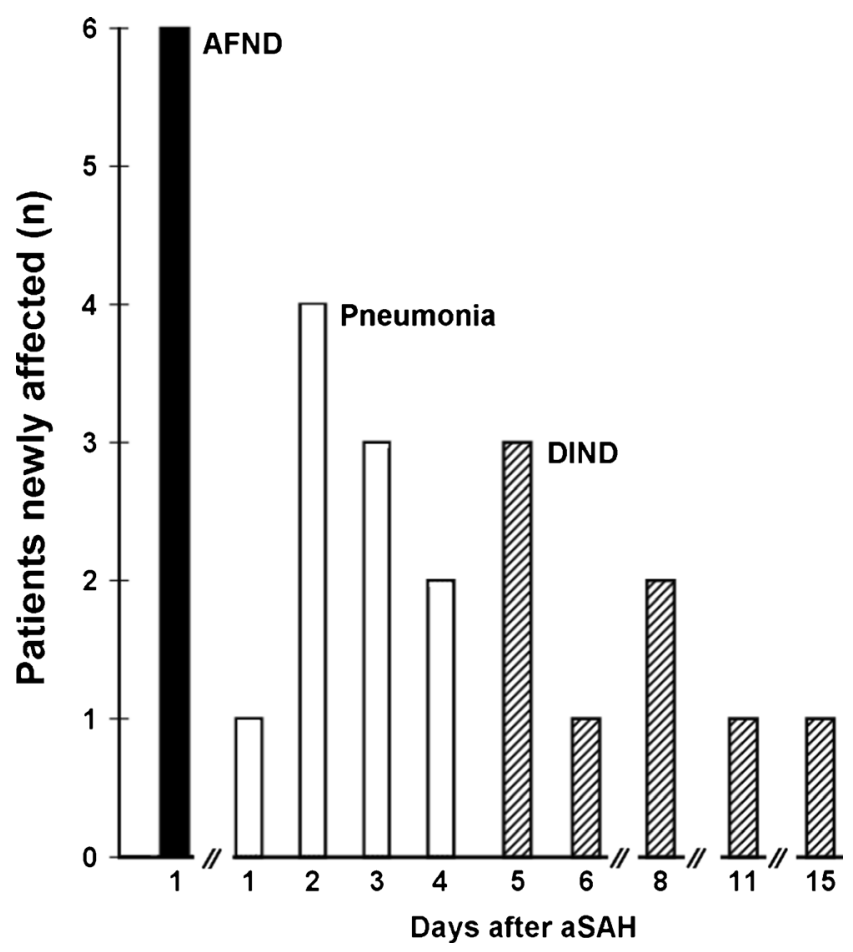

Fig. 1 Time course of adverse events. Six patients (33\%) presented acute focal neurological deficits (AFNDs). Ten patients (56\%) suffered from early onset pneumonia (onset day median $=2.5$; range $=1-4$ ). Eight patients $(45 \%)$ developed DIND (onset day median $=7$; range $=5-15$ )

\section{Cerebral Metabolism}

Levels of cerebral glucose, pyruvate, and glycerol were comparable between patients with/without AFND, DIND, and bacterial pneumonia in this patient population. The lactate/ pyruvate ratio (LPR) appeared to be elevated in DIND patients (days 5-7), but reached no significant difference (Fig. 2). The time pattern of lactate elevations in patient with pneumonia and DIND matched the timing of their respective onset days, while levels of lactate in patients with/without AFND were comparable.

\section{Cerebral Lactate}

Mean cerebral lactate levels were elevated at day 3 in patients with bacterial pneumonia $(n=9$, median $=6.82 \mathrm{mmol} / \mathrm{L})$ vs. patient without bacterial pneumonia $(n=6$, median $=$ $2.90 \mathrm{mmol} / \mathrm{L}$ ). Intergroup difference (Mann-Whitney $U$ test) was significant $p=0.036$ (Fig. 2). Lactate at day 7 was significantly higher in patients fulfilling criteria for DIND $(n=6$, median $=8.16 \mathrm{mmol} / \mathrm{l}$ ) compared to neurologically asymptomatic patients $(n=10$, median $=5.85 \mathrm{mmol} / 1, p<0.05$, Fig. 3 ).

Comparisons of Adverse Event's Onsets and Peaks of the Lactate Levels

The onset of pneumonia was at median 2.5 days $(60 \mathrm{~h}$, range 24-192 h) after aSAH onset. To compare timing of lactate peaks and more detailed pneumonia onset, we used hourly measured values. Significantly elevated 


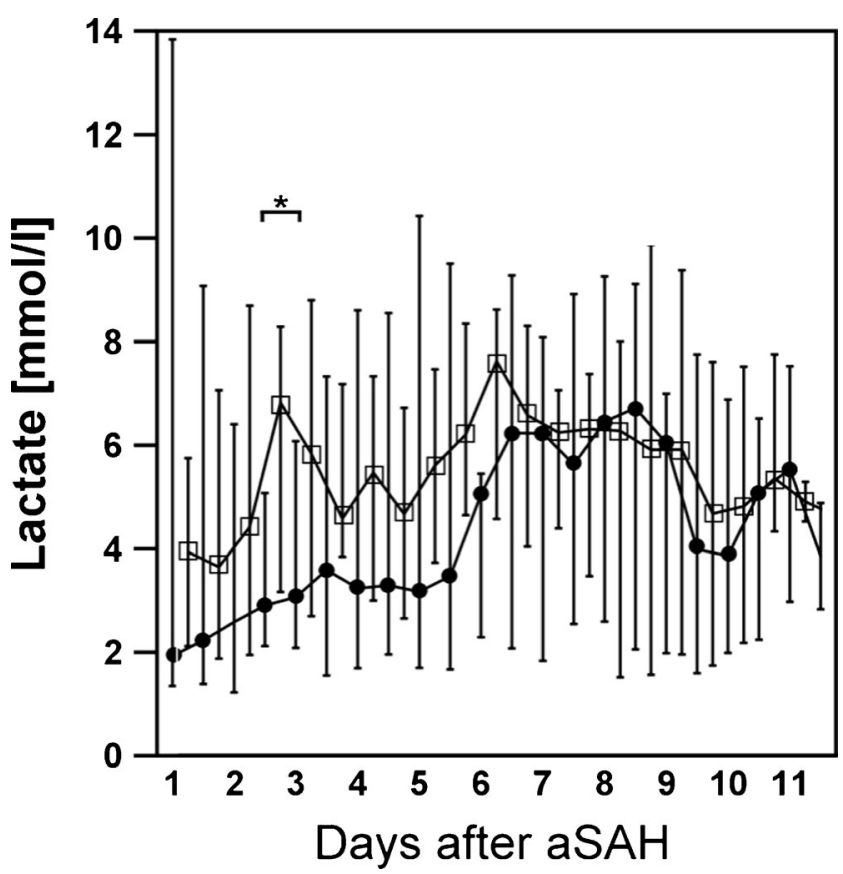

Fig. 2 Time course of cerebral lactate in aSAH patients with pneumonia. Lactate was elevated at day 3 (peak $61-72 \mathrm{~h}$ after onset) in patients with infections (squares, $n=9$, median $=6.82 \mathrm{mmol} / \mathrm{L}$ ) vs. patient without infections (circles, $n=6$, median $=2.90 \mathrm{mmol} / \mathrm{L}$ ). Values are given as means; \pm 1 SD. SD, standard deviation. ${ }^{*}$ Intergroup difference in MannWhitney $U$ test was significant $(p=0.036)$

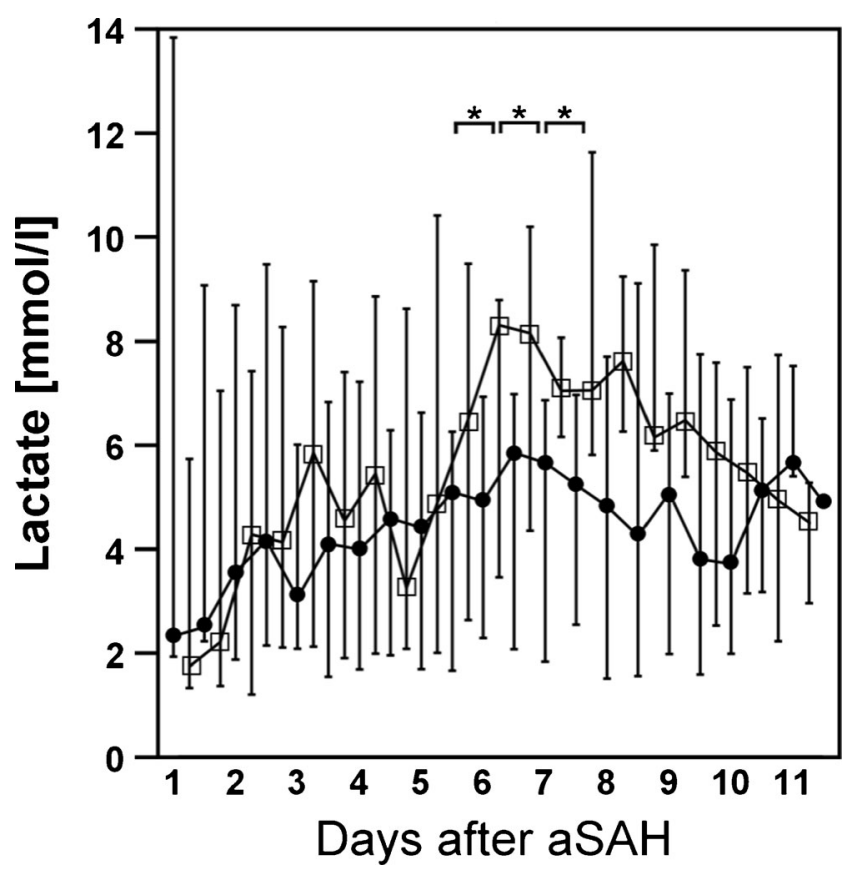

Fig. 3 Time course of cerebral lactate in patients with DIND. Lactate at day 7 (peak hour $145-156$ after onset) was significant $(* p<0.05$ ) higher in patients fulfilling criteria for DIND (squares $n=6$, median= $8.16 \mathrm{mmol} / \mathrm{l}$ ) compared to neurologically asymptomatic patients (circles $n=10$, median $=5.85 \mathrm{mmol} / \mathrm{l})$. Values are given as means; $\pm 1 \mathrm{SD}$. SD standard deviation lactate levels have been found at median $84 \mathrm{~h}$, range $42-139 \mathrm{~h}$ after aSAH. The early peak of lactate levels was within $61-$ $72 \mathrm{~h}$ after aSAH onset, meeting the median of pneumonia onset perfectly. DIND was diagnosed at median day 7 (median $168 \mathrm{~h}$, range $120-360 \mathrm{~h}$ ) after aSAH onset, having significant higher lactate levels at similar time (median $150 \mathrm{~h}$; range 128 199).

Multivariate Analysis

Multivariate analysis for levels of lactate (days 2-4) was calculated for multiple factors as independent variables: pneumonia nolyes, Hunt and Hess grade low/high, AFND no/yes, and coiling vs clipping. Levene's tests of equality of error variances were performed firstly for each day to test the assumption of MANOVA that the variances of each variable are equal across the groups.

The tests were not significant, indicating that difference of variances in MANOVA can be interpreted. It turned out that pneumonia was the only significant factor. All other independent factors included in the model were not able to explain the variance of lactate levels at the relevant days. Pillai's trace-based $p$ value was significant $(F$ value $=7.33$ with exact testing; partial eta squared $=0.846 ; p=0.042$ ).

The same multivariate analysis has been performed for levels of lactate at days 8-11 with factors DIND yes /no, Hunt and Hess grade low/high, AFND no/yes, and coiling vs clipping. Only DIND was a significant factor $(F$ value $=$ 249.875; partial eta squared $=0.996 ; p=0.040$ ), while all other independent variables included in the model failed to explain differences in variance of lactate levels.

\section{Receiver Operation Characteristic}

To determine cut-off values of cerebral lactate predicting pneumonia, and DIND, a ROC analysis was carried out. For pneumonia at day 3 , the area under curve (AUC) was 0.833 with $p=0.034$. The optimum cut-off point to predict pneumonia was $3.57 \mathrm{mmol} / \mathrm{L}$ with a sensitivity of 0.77 and a specificity of 0.66 . Cerebral lactate $>6.28 \mathrm{mmol} / \mathrm{L}$ at day 8 turned out to be also predictive for DIND (AUC $=0.875 ;$ sensitivity/specificity $=0.75 / 0.62 \% ; p=$ 0.042). Positive and negative predictive values have not been calculated due to small sample size. Lactate failed to be predictive for mortality during monitoring (until day 15), and follow up (day 180).

\section{Discussion}

The aim of the present study was to measure parameters of cerebral metabolism in the ECF of aSAH patients and to 
evaluate its potential value reflecting primary aSAH severity and occurrence of infection development, mainly bacterial pneumonia. To our knowledge, this study is the first detailed investigation on cerebral interstitial energy metabolism in aSAH patients in relation to infection. The idea behind it is that the primary damage may initiate inflammatory processes leading to a depressed immune status and more likely infections after aSAH.

\section{Brain Metabolism}

Cerebral MD provides information on the metabolic state of the injured brain, and it permits to determine a variety of markers of immunocompetence in the cerebral ECF [26]. This is of relevance since both the blood-brain and blood-CSF barriers may be variably disrupted following aSAH, rendering blood and CSF levels of such markers less reliable [27]. Our most interesting finding is that early elevated lactate levels in ECF correlated in univariate and multivariate analysis with occurrence of bacterial pneumonia, while late elevations correlated with DIND after aSAH. The causality of elevated cerebral lactate in patients with pneumonia remains unclear; besides being a marker of the severity of compromised cerebral tissue after aSAH, systemic hypoxemia, or lactatemia with damaged blood brain barrier are possible explanations for high-cerebral lactate levels. Our second finding, that a second peak of cerebral lactate is related to the occurrence of DIND confirms previous data by others [28] and our group [22]. These studies have identified cerebral lactate as well as the lactate/pyruvate ratio to be predictive for cerebral ischemia in aSAH patients $[28,29]$.

This is in line with our results concerning the lactate/ pyruvate ratio which appeared to be elevated in DIND patients in the same time period (days 5-7), but reached no significant difference probably due to interindividual variability, lowsample size, and the grade of ischemia present in these patients (Fig. 4).

\section{Aneurysmal SAH-Induced Bacterial Pneumonia}

Bacterial pneumonia is not only the leading cause of severe sepsis in ICU patients and the most common cause of death in stroke patients, but also a well-known complication in aSAH $[2,3,6]$. The high incidences of abnormal mental status, reduction of bulbar reflexes, dysphagia, and aspiration or mechanical ventilation for several days are considered to be major contributors to the high incidence of bacterial pneumonia after stroke [30]. It is therefore not surprising that this complication is frequent in high-grade SAH patients (WFNS grade IV-V) [6]. Experimental and clinical studies stress the role of immunocompetence and suggest that dysphagia is a confounder rather than the sole reason for nosocomial infections $[4,5]$. This is supported by experimental findings in the mouse model of transient middle cerebral artery occlusion, demonstrating that even 14 days after cerebral ischemia intranasal application of only 200 colony-forming units of Streptococcus pneumoniae was sufficient to cause severe pneumonia and bacteremia in stroke animals, while in contrast, 200,000 colony-forming units were needed to induce pneumonia of similar severity in sham-operated animals [31]. This means that otherwise harmless minor bacterial aspiration leads to severe pneumonia and bacteremia after ischemic stroke suggesting that the deleterious combination of stroke-induced immunodeficiency with stroke-facilitated aspiration dramatically increases the susceptibility to infection. Thus, immunodepression after acute CNS injury appears to be an important risk factor for the development of infectious complications and outcomes.

A major limitation to our study is the small number of patients. Furthermore, SAH is a heterogeneous disease limiting the generalization of our results. These patients are commonly intubated and at high risk for aspiration, pulmonary edema, and fluid overload, all of which increase the chances of pneumonia [32]. Interestingly, in our study population, the median duration of mechanical ventilation was comparable in patients with and without pneumonia.
Fig. 4 Time course of the lactate/ pyruvate ratio (LPR) after aSAH. After a short peak of the LPR in no-DIND-patients (day1) possibly an insertion effectlevels appeared to be higher in DIND patients (NS)

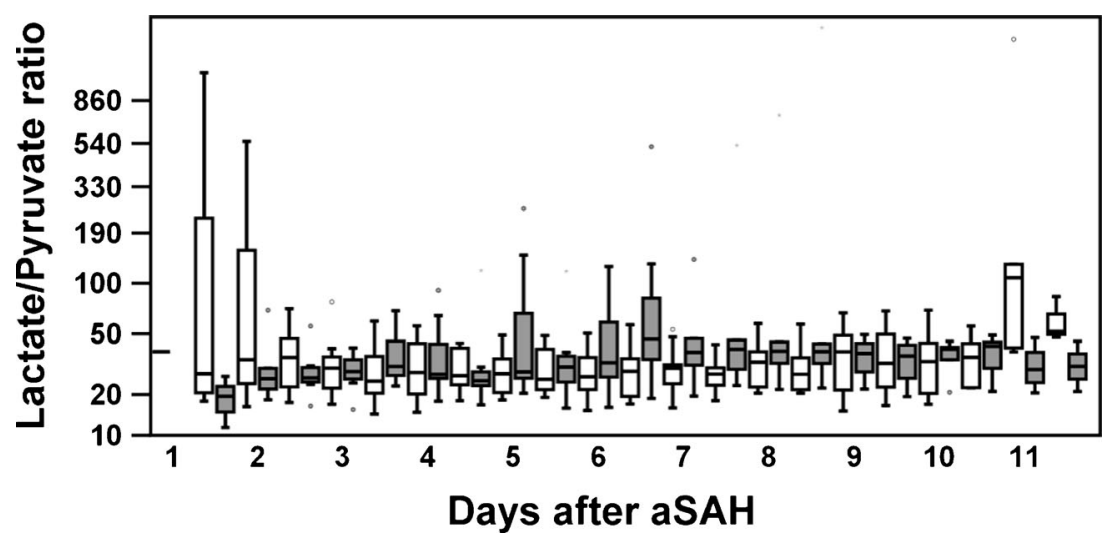


Microdialysis is a local monitor of cerebral metabolism and results cannot be generalized for the whole brain. Nevertheless, due to the time and cost intensive nature of microdialysis, only a few data are available in prospective studies in aSAH patients. The technique can detect harmful secondary insults, and may be used as end points for goal-directed therapy, with the aim of creating an optimal physiological environment for the comatose-injured brain [32]. A higher patient number would allow more substantial analysis to confirm that early lactate levels (day 3 ) serve as predictor of pneumonia. This might support the hypothesis that early primary brain damage due to an aSAH, reflected in high-lactate levels, initiates inflammatory processes and immunosuppression-followed by bacterial infections later on.

\section{Conclusion}

Early elevated lactate levels in ECF were correlated with occurrence of bacterial pneumonia, while late elevations correlated with DIND after aSAH. The causality of elevated lactate remains unclear in patients with pneumonia: a systemic hypoxemia or lactatemia with damaged blood brain barrier are possible reasons. Thus, in a next step, a more comprehensive analysis will be performed to elucidate possible relationships between MD markers and markers of immunocompetence. MD may help to identify aSAH patients at risk for severe infections, probably profiting from earlier therapy.

Acknowledgments This study is supported by grants from the Deutsche Forschungsgemeinschaft (DFG DR 323/5-1) and the Bundesministerium für Bildung und Forschung (Center for Stroke Research Berlin, 01 EO 0801; Bernstein Center for Computational Neuroscience Berlin 01GQ1001C B2) to Dr. Dreier.

Conflict of Interest The authors declare that they have no conflict of interest.

\section{References}

1. Oddo M, Levine JM, Frangos S, et al. Brain lactate metabolism in humans with subarachnoid hemorrhage. Stroke. 2012;43:1418-21.

2. Hilker R, Poetter C, Findeisen N, et al. Nosocomial pneumonia after acute stroke: implications for neurological intensive care medicine. Stroke. 2003;34:975-81.

3. Frontera JA, Fernandez A, et al. Impact of nosocomial infectious complications after subarachnoid hemorrhage. Neurosurgery. 2008;62:80-7.

4. Dirnagl U, Klehmet J, Braun JS, et al. Stroke-induced immunodepression: experimental evidence and clinical relevance. Stroke. 2007;38:770-3.

5. Harms H, Prass K, Meisel C, et al. Preventive antibacterial therapy in acute ischemic stroke: a randomized controlled trial. PloS One. 2008;3:2158.
6. Sarrafzadeh A, Schlenk F, Meisel A, et al. Immunodepression after aneurysmal subarachnoid hemorrhage. Stroke. 2011;42:53-8.

7. Sehba FA, Pluta R, et al. Metamorphosis of subarachnoid hemorrhage research: from delayed vasospasm to early brain injury. Mol Neurobiol. 2011;43:27-40.

8. Chaichana KL, Pradilla G, Huang J, Tamargo RJ. Role of inflammation (leukocyte-endothelial cell interactions) in vasospasm after subarachnoid hemorrhage. World Neurosurg. 2010;73:22-41.

9. Sercombe R, Dinh YR, Gomis P. Cerebrovascular inflammation following subarachnoid hemorrhage. Jpn J Pharmacol. 2002;88: 227-49.

10. Sarrafzadeh AS, Nagel A, Czabanka M, et al. Imaging of hypoxicischemic penumbra with (18)F-fluoromisonidazole PET/CT and measurement of related cerebral metabolism in aneurysmal subarachnoid hemorrhage. J Cereb Blood Flow. 2010;30:36-45.

11. Mathiesen T, Andersson B, Loftenius A, et al. Increased interleukin-6 levels in cerebrospinal fluid following subarachnoid hemorrhage. $\mathrm{J}$ Neurosurg. 1993;78:562-7.

12. Osuka K, Suzuki Y, Tanazawa T, et al. Interleukin-6 and development of vasospasm after subarachnoid haemorrhage. Acta Neurochir. 1998;140:943-51.

13. Schoch B, Regel JP, Wichert M, et al. Analysis of intrathecal interleukin-6 as a potential predictive factor for vasospasm in subarachnoid hemorrhage. Neurosurgery. 2007;60:828-36. discussion 828-836.

14. Fassbender K, Hodapp B, Rossol S, et al. Inflammatory cytokines in subarachnoid haemorrhage: association with abnormal blood flow velocities in basal cerebral arteries. J Neurol Neurosurg Psychiatry. 2001;70:534-7.

15. Graetz D, Nagel A, et al. High ICP as trigger of proinflammatory IL-6 cytokine activation in aneurysmal subarachnoid hemorrhage. Neurol Res. 2009;32:728-35.

16. Schneider UC, Schiffler J, et al. Functional analysis of proinflammatory properties within the cerebrospinal fluid after subarachnoid hemorrhage in vivo and in vitro. J Neuroinflammation. 2012;9:28.

17. Teasdale GM, Drake CG, Hunt W, Kassell N, et al. A universal subarachnoid hemorrhage scale: report of a committee of the World Federation of Neurosurgical Societies. J Neurol Neurosurg Psych. 1988;51:1457.

18. Hunt WE, Hess RM. Surgical risk as related to time of intervention in the repair of intracranial aneurysms. J Neurosurg. 1968;28:14-20.

19. Fisher CM, Kistler JP, Davis JM. Relation of cerebral vasospasm to subarachnoid hemorrhage visualized by computerized tomographic scanning. Neurosurgery. 1980;6:1-9.

20. Vergouwen MDI, Vermeulen M, van Gijn J, et al. Definition of delayed cerebral ischemia after aneurysmal subarachnoid hemorrhage as an outcome event in clinical trials and observational studies: proposal of a multidisciplinary research group. Stroke. 2010;41: 2391-5.

21. Dreier JP, Major S, Pannek H-W, et al. Spreading convulsions, spreading depolarization and epileptogenesis in human cerebral cortex. Brain J Neurol. 2012;135:259-75.

22. Sarrafzadeh A, Haux D, Plotkin M, et al. Bedside microdialysis reflects dysfunction of cerebral energy metabolism in patients with aneurysmal subarachnoid hemorrhage as confirmed by $15 \mathrm{O}-\mathrm{H} 2 \mathrm{O}$-PET and 18 F-FDG-PET. J Neuroradiol. 2005;32:348-51.

23. Drenckhahn C, Brabetz C, Major S, et al. Criteria for the diagnosis of noninfectious and infectious complications after aneurysmal subarachnoid hemorrhage in DISCHARGE-1. Acta Neurochir Suppl. 2013;115:153-9.

24. Pillai KC. Some new test criteria in multivariate analysis. Ann Math Statist. 1955;26:117-21. 
25. Olson C. Comparative robustness of six tests in multivariate analysis of variance. J Am Stat Assoc. 1974;69:894-908.

26. Hillman J, Aneman O, Persson M, et al. Variations in the response of interleukins in neurosurgical intensive care patients monitored using intracerebral microdialysis. J Neurosurg. 2007;106: $820-5$.

27. Hanafy KA, Grobelny B, Fernandez L, et al. Brain interstitial fluid TNF-alpha after subarachnoid hemorrhage. J Neurol Sci. 2010;291: 69-73.

28. Goodman JC, Valadka AB, Gopinath SP, et al. Extracellular lactate and glucose alterations in the brain after head injury measured by microdialysis. Crit Care Med. 1999;27:1965-73.
29. Nilsson OG, Brandt L, Ungerstedt U, Säveland H. Bedside detection of brain ischemia using intracerebral microdialysis: subarachnoid hemorrhage and delayed ischemic deterioration. Neurosurgery. 1999;45(5):1176-84.

30. Perry L, Love CP. Screening for dysphagia and aspiration in acute stroke: a systematic review. Dysphagia. 2001;16:7-18.

31. Prass K, Braun JS, Dirnagl U, Meisel C, et al. Stroke propagates bacterial aspiration to pneumonia in a model of cerebral ischemia. Stroke. 2006;37:2607-12.

32. Komotar RJ, Schmidt JM, Starke RM, et al. Resuscitation and critical care of poor-grade subarachnoid hemorrhage. Neurosurgery. 2009;64:397-410. 